\title{
EFEITOS DO TREINAMENTO FÍSICO NO ENDOTÉLIO APÓS CIRURGIA DE REVASCULARIZAÇÃO
}

\author{
EFFECTS OF PHYSICAL TRAINING ON ENDOTHELIUM AFTER CORONARY ARTERY BYPASS GRAFT \\ EFECTOS DEL ENTRENAMIENTO FÍSICO EN EL ENDOTELIO DESPUÉS DE REVASCULARIZACIÓN
}

Original Articie

ARTIGO ORIGINAL ARTíCULO ORIGINAL
Priscila Aikawa' (Fisioterapeuta) Luis Ulisses Signori² (Fisioterapeuta) Melina Hauck' (Fisioterapeuta) Ana Paula Cardoso Pereira' (Fisioterapeuta)

Renata Gomes Paulitsch (Nutricionista)

Claudio Tafarel Mackmillan da Silva ${ }^{3}$ (Graduando de Fisioterapia) William Peres ${ }^{4}$ (Farmacêutico) Felipe da Silva Paulitsch' (Médico Cardiologista)

1. Universidade Federal do Rio Grande, Rio Grande, RS, Brasil. 2. Universidade Federal de Santa Maria, Santa Maria, RS, Brasil. 3. Faculdade Anhanguera do Rio Grande, Rio Grande, RS, Brasil. 4. Universidade Federal de Pelotas, Pelotas, RS, Brasil.

\section{Correspondência:}

Universidade Federal do Rio Grande (FURG), Instituto de Ciências Biológicas (ICB). Rio Grande, Rio Grande, RS, Brasil. Av. Itália km 8, Campus Carreiros. 96.201-900. Rio Grande, RS, Brasil. priaikawa@hotmail.com; priscila.aikawa@furg.br

\section{RESUMO}

Introdução: A cirurgia de revascularização do miocárdio (CRM) é uma das mais frequentes cirurgias realizadas em todo o mundo, muitos estudos vêm relatando os benefícios do treinamento físico para a melhora da capacidade funcional, porém há poucos estudos sobre os efeitos na função endotelial vascular. Objetivo: Analisar os efeitos do treinamento físico sobre a função endotelial vascular em pacientes submetidos à CRM, isoladamente na fase tardia da reabilitação após seis meses de programa de reabilitação cardíaca (PCR). Métodos: Foram incluídos pacientes que haviam sido submetidos à CRM no período máximo de um ano após a cirurgia. O PRC foi empregado durante seis meses consecutivos, com três sessões semanais. Todos os pacientes realizaram exames laboratoriais, teste de força muscular de uma repetição máxima (1-RM) para os membros superiores e inferiores, teste de caminhada de 6 min (TC6M) e avaliação da função endotelial através da técnica de vasodilatação mediada pelo fluxo. Resultados: Onze pacientes iniciaram o PRC, porém nove pacientes o completaram. A média de idade foi de 66 anos (50 a 82 anos) e o sexo masculino foi predominante $(55,6 \%)$. Houve mudanças significativas nos exames laboratoriais bioquímicos: aumento do colesterol total (Basal: 162 $\pm 31 \mathrm{mg} / \mathrm{dL}$ vs. $195 \pm 39 \mathrm{mg} / \mathrm{dL} ; \mathrm{P}=0,012$ ) e diminuição da hemoglobina glicada (Basal: 6,74 $\pm 1,64 \%$ vs. 6,26 \pm $1,62 \% ; P=0,028)$. A força muscular aumentou significativamente nos membros superiores e inferiores $(P=0,030$ e $\mathrm{P}=0,038$, respectivamente); no TC6M observou-se um aumento significativo de $20 \%$ na distância percorrida $(P=0,020)$ após seis meses consecutivos de treinamento e houve uma melhora na vasodilatação mediada pelo fluxo (Basal: 6,35 $\pm 3,92 \%$ vs. 6 meses: 9,90 $\pm 4,19 \% ; P=0,026$ ). Conclusão: $O$ treinamento combinado realizado em seis meses ajudou a melhorar a função endotelial e a capacidade funcional de pacientes sedentários que foram submetidos à CRM na fase tardia da reabilitação.

Palavras-chave: exercício, endotélio vascular, ponte de artéria coronária, revascularização miocárdica.

\section{ABSTRACT}

Introduction: The coronary artery bypass graft surgery $(C A B G)$ is one of the most common surgeries performed worldwide, many studies have reported the benefits of physical training to improve functional capacity, however there are few studies about the effect of this training on vascular endothelial function. Objective: To analyze the effects of physical training on vascular endothelial function in patients who underwent $C A B G$ surgery alone in the last phase of rehabilitation after six months of cardiac rehabilitation program (CRP). Methods: Patients who underwent $C A B G$ surgery in a maximum period of one year after surgery were included in the study. The CRP was carried out during six consecutive months, with three sessions per week. All patients underwent biochemical blood tests, muscle strength testing of one repetition maximum (1-RM test) for upper and lower limbs, 6-minute walk test (6MWT), and evaluation of endothelial function through the flow-mediated vasodilation technique. Results: Eleven patients started CRP, but only nine patients completed it. The mean age was 66 years old (50 to 82 years old), males being predominant (55.6\%). There were significant changes in biochemical laboratory tests: increase on total cholesterol (Baseline: $162 \pm 31 \mathrm{mg} / \mathrm{dL}$ vs. $195 \pm 39 \mathrm{mg} / \mathrm{dL} ; P=0.012$ ), and decrease on glycated hemoglobin (Baseline: $6.74 \pm$ $1.64 \%$ vs. $6.26 \pm 1.62 \% ; P=0.028$ ). Muscle strength was significantly increased in upper and lower limbs ( $P=0.030$, and $P=0.038$, respectively) and in 6 MWT we observed a significant increase of $20 \%$ in distance traveled $(P=0.020)$ after six consecutive months of training. There was also a flow-mediated vasodilation improvement $(6.35 \pm 3.92 \% \mathrm{vs.} 9.90 \pm$ 4.19\%; $P=0.026)$. Conclusion: The combined training carried out in six months helped to improve endothelial function and functional capacity in sedentary patients submitted to $C A B G$ surgery in the last phase of cardiac rehabilitation.

Keywords: exercise, endothelium vascular, coronary artery bypass, myocardial revascularization.

\section{RESUMEN}

Introducción: La cirugía de revascularización miocárdica (CRM) es una de las cirugías más comunes que se realizan en todo el mundo, muchos estudios han reportado los beneficios del entrenamiento físico para mejorar la capacidad funcional, pero hay pocos estudios sobre el efecto en la función endotelial vascular. Objetivo: Analizar los efectos del entrenamiento físico sobre la función endotelial vascular en los pacientes sometidos a CRM solo en la última fase de la rehabilitación después de seis meses del programa de rehabilitación cardíaca (PRC). Métodos: Fueron incluidos los pacientes que habían sido sometidos a CRM con el período máximo de un año después de la 
cirugía. Los pacientes fueron sometidos a lo PRC por seis meses consecutivos, con tres sesiones semanales. Todos los pacientes realizaron pruebas de laboratorio, pruebas de fuerza muscular una repetición máxima (1-RM) para miembros superiores e inferiores, prueba de la marcha de 6 minutos (PM6M) y la evaluación de la función endotelial mediante la técnica de la vasodilatación mediada por flujo. Resultados: Once pacientes iniciaron el PRC, pero nueve pacientes lo completaron. La edad promedio fue de 66 años (50-82 años) y los varones predominaron (55,6\%). Hubo cambios significativos en las pruebas de laboratorio bioquímico: aumento en el colesterol total (basal: $162 \pm 31 \mathrm{mg}$ / dl vs. $195 \pm 39 \mathrm{mg} / \mathrm{dl} ; p=0,012$ ) y disminución de la hemoglobina glucosada (basal: 6,74 $\pm 1,64 \%$ \% vs. 6,26 $\pm 1,62$, $p=0,028)$. La fuerza muscular se incrementó significativamente en los miembros superiores e inferiores $(P=0,030$ y $P=0,038$, respectivamente) y en $P M 6 M$ se observó un aumento significativo de $20 \%$ en la distancia cubierta ( $P$ $=0,020$ ) después de seis meses consecutivos de entrenamiento y también la vasodilatación mediada por flujo ha mejorado (basal: 6,35 $\pm 3,92 \%$ vs. 9,90 $\pm 4,19 \%, p=0,026$ ). Conclusión: El entrenamiento combinado realizado en seis meses ha mejorado la función endotelial y la capacidad funcional de los pacientes sedentarios sometidos a CRM en la última fase de la rehabilitación.

Palabras clave: ejercicio, endotelio vascular, puente de arteria coronaria, revascularización miocárdica.

\section{INTRODUÇÃO}

A reabilitação cardíaca melhora a capacidade física, função psicológica e social dos pacientes, além de estabilizar, retardar ou ainda reverter a progressão do processo da doença aterosclerótica, reduzindo a morbidade e a mortalidade de pacientes cardiopatas. São candidatos elegíveis para os serviços de reabilitação cardíaca: pacientes com doença coronária suspeita ou comprovada insuficiência cardíaca, transplante cardíaco, doença vascular periférica e outras doenças cardiovasculares ${ }^{1,2}$.

Recentemente, ensaios clínicos randomizados relataram a eficácia de programas de reabilitação cardíaca (PRC) independentemente do formato dos exercícios: aeróbicos, resistidos ou combinados em pacientes com doenças coronarianas $5^{3,4}$; ainda em outros estudos foram investigados os efeitos do PRC sobre a função vascular endotelial em pacientes com doença coronariana estável $\left.\right|^{5}$, doença cardíaca isquêmi$\mathrm{ca}^{6}$, infarto agudo do miocárdio e doença cardíaca crônica ${ }^{3,7,8}$, porém não há estudos que investigaram a função vascular endotelial em pacientes que participaram de PRC após recente CRM.

A detecção de disfunção endotelial arterial avaliada pela vasodilatação mediada pelo fluxo é um preditor de eventos adversos cardiovasculares à longo prazo em indivíduos saudáveis ${ }^{9}$ e é um dado adicional importante para o prognóstico das doenças cardiovasculares, além dos fatores de risco tradicionais ${ }^{10}$.

A reabilitação cardíaca através do treinamento físico está associada com a melhora da função endotelial, estresse oxidativo e inflamação ${ }^{11,12}$, além de promover efeitos benéficos sobre os desfechos cardiovasculares e diminuir as internações hospitalares e a mortalidade ${ }^{13,14}$.

Portanto, o objetivo deste estudo é investigar os efeitos de um programa de treinamento de reabilitação convencional com exercícios combinados (aeróbicos e resistidos) sobre a função endotelial vascular em pacientes que foram submetidos à CRM na fase III da reabilitação.

\section{CASUÍSTICA E MÉTODOS}

Este estudo foi aprovado pelo Comitê de Ética Local do Hospital da Associação de Caridade Santa Casa do Rio Grande, Rio Grande, RS, Brasil, com número de protocolo 003/2013, registrado em ClinicalTrials com identificador NCT02265107 e realizado em consonância com a Resolução 466/12 do Conselho Nacional de Saúde. Trata-se de um estudo clínico prospectivo com intervenção terapêutica. Todos os pacientes que aceitaram participar do estudo assinaram um Termo de Consentimento Livre e Esclarecido antes de iniciar o PRC. Foram incluídos neste estudo os pacientes que buscaram o atendimento do PRC na clínica-escola de Fisioterapia da Faculdade Anhanguera do Rio
Grande, Rio Grande, R.S., Brasil; que haviam realizado a CRM no Hospital de Cardiologia Doutor Pedro Bertoni da Associação de Caridade Santa Casa do Rio Grande, Rio Grande, RS, Brasil, com um período de seis meses a um ano de cirurgia e foram excluídos os pacientes que haviam realizado outros procedimentos cirúrgicos cardiológicos associados à CRM. Todos os pacientes foram avaliados por um medico cardiologista e classificados de acordo com a Classificação Funcional da New York Heart Association (NYHA) como classes I ou II.

Inicialmente, 11 pacientes aceitaram realizar a primeira avaliação e todos atendiam os critérios de inclusão, mas somente nove pacientes completaram o PRC de seis meses de duração, sendo os motivos das desistências: (1) pessoal e (2) infecção respiratória com necessidade de internação hospitalar.

O PRC foi empregado por seis meses consecutivos com três sessões semanais de 90 minutos de duração. O programa de treinamento combinado consistia em exercícios aeróbicos em esteira e bicicleta ergométrica e exercícios resistidos de membros superiores e inferiores. Cada sessão incluía: (1) Cinco min de aquecimento com alongamentos leves de grandes cadeias musculares; (2) 30 min de exercícios aeróbicos sem pausa (alternando $15 \mathrm{~min}$ em esteira ergométrica mais $15 \mathrm{~min}$ em bicicleta ergométrica); (3) 20 min de exercícios resistidos com halteres para membros superiores e tornozeleiras para membros inferiores realizados em diagonais (primitiva e funcional) da técnica do Kabat que consistia em seis séries de dez repetições para membros superiores (60 repetições ao todo) e inferiores (60 repetições ao todo) e (4) cinco min de relaxamento final com alongamentos leves.

Inicialmente, no treinamento aeróbico foi limitada a frequência cardíaca (FC) máxima estabelecida entre 30-40\% da FC em repouso devido todos os pacientes serem sedentários e passarem recentemente pelo procedimento cirúrgico e os exercícios resistidos com carga de 30-40\% da carga aferida no teste de uma repetição máxima (1-RM) e limitados aos sintomas relatados pelos pacientes. Gradativamente, o treinamento aeróbico e a carga dos exercícios foram aumentados para que até o final do programa os pacientes atingissem $60 \%$ da FC em repouso, $60 \%$ da carga do teste de 1-RM e a intensidade e velocidade do treinamento aeróbico aumentadas também de acordo com os sintomas relatados pelos pacientes, além de realizar os exercícios sem compensações no tronco, sem queixas de dores e fadiga musculares.

A frequência cardíaca, a frequência respiratória e a saturação do oxigênio pela oximetria de pulso (\%) foram aferidas no início, meio e fim de cada sessão diária. 
Na avaliação basal realizada antes do início do PRC foram realizados exames bioquímicos laboratoriais de sangue [glicemia, insulina, hemoglobina glicada, colesterol total, colesterol de alta densidade $(H D L)$, colesterol de baixa densidade (LDL), triglicerídeos]; índice de massa corpórea (IMC); força muscular dos membros superiores (músculo bíceps braquial) e dos membros inferiores (músculo quadríceps) através do teste de 1-RM; avaliação da capacidade funcional através do teste de caminhada dos seis minutos e avaliação da função endotelial vascular através da vasodilatação mediada pelo fluxo (flow mediated-vasodilation - FMD).

Colesterol, triglicerídeos, HDL e glicose na urina foram medidas usando kits comerciais LAB TEST (Lagoa Santa, MG, Brasil) e analisados no equipamento $\angle A B M A X 240^{\circledR}$ (Tokyo, Japan). O LDL foi calculado pela fórmula de Friedewald. A glicemia foi medida através de ensaio Trinder (calorimetria) no equipamento $\angle A B M A X 240^{\circledR}$ (Tokyo, Japan). A insulina foi quantificada pelo método de imunofluorescência com o equipamento Immulite ${ }^{\circledR}$ DPC (Diagnostic Products Corporation - DPC, Los Angeles, CA, USA). A hemoglobina glicada $\left(\mathrm{HbA}_{1 \mathrm{C}}\right)$ foi quantificada pelo método enzimático com o equipamento LAB MAX 240 ${ }^{\circledR}$ (Tokyo, Japan).

O teste de caminhada dos seis minutos (TC6M) foi utilizado para avaliar a capacidade funcional dos pacientes, o qual é extremamente importante para avaliar a capacidade física e a tolerância ao exercício para a avaliação clínica e o prognóstico de pacientes com doenças cardiovasculares e pulmonares ${ }^{15,16}$. OTC6M foi realizado no primeiro dia da avaliação em um corredor linear com 40 metros de comprimento. Durante o teste, o pesquisador dava comandos verbais ao paciente para que caminhasse em maior velocidade possível suportada por ele e o pesquisador perguntava durante todo o teste as sensações físicas que o paciente estava sentindo. A frequência cardíaca, frequência respiratória e a saturação do oxigênio pela oximetria de pulso (Nonin ${ }^{\circledR}$, model 9500, Plymouth, USA), foram aferidas no início do teste, no terceiro minuto e ao final do teste; e a pressão arterial sistêmica foi aferido antes e ao final do teste através de um esfignomanômetro manual calibrado esfigmomanômetro manual (BD - Becton Dickinson, Indústrias Cirúrgicas Ltda. São Paulo, SP, Brasil) e estetoscópio Littmann Classic $\|^{\circledR}$.

A captura da profundidade e das imagens foi mantida constante ao longo do estudo. A técnica de avaliação através vasodilatação mediada pelo fluxo, conhecida pela sigla FMD (Flow-mediated vasodilation), de acordo com o Guidelines for the ultrasound assessment of endotelial- dependet FMD of the brachial artery do Journal of the American Collegge of Cardiology ${ }^{17}$ quantifica a variação do diâmetro da artéria braquial após 60 segundos de hiperemia reativa, ou seja, após desinsuflação do manguito colocado ao redor do braço e inflado a $50 \mathrm{mmHg}$ acima da pressão arterial sistólica durante cinco minutos. Para avaliar o fluxo sanguíneo basal e fluxo imediatamente após a liberação do manguito foi obtida, após no máximo 15 segundos da desinsulflação do manguito, os sinais pulsáteis da velocidade captada pelo Ultrassom Doppler (avaliada utilizando um ângulo $\leq 60^{\circ}$ entre o cabeçote e o vaso). A vasodilatação independente do endotélio (nitroglycerin-mediated dilation [NMD]) é avaliada com o uso de nitroglicerina $(0,4 \mathrm{mg})$ sublingual. $O$ aumento do diâmetro após uma pulverização de nitroglicerina sublingual $(0,4 \mathrm{mg})$ foi usado como uma medição de vasodilatação independente do endotélio. As respostas do diâmetro do vaso à hiperemia reativa e à nitroglicerina foram expressas como a porcentagem da mudança em relação ao diâmetro imediatamente antes da inflação do manguito e diâmetro imediatamente antes da administração de drogas. As medições de diâmetro da artéria braquial foram realizadas por dois avaliadores independentes, utilizando um sistema de análise quantitativo após as intervenções antes e ao final do PRC. O segundo avaliador ao realizar as medidas desconhecia as medidas aferidas pelo primeiro avaliador. Diferenças maiores que 0,01 mm entre avaliadores (média de diâmetro do vaso) foram repetidas. Todos os dados foram mensurados duas vezes, e os valores finais foram apresentados como média \pm desvio padrão.

Todos os testes foram repetidos após seis meses de treinamento e comparados com a avaliação basal. O programa de treinamento foi finalizado após esta avaliação de seis meses e os pacientes foram orientados a seguir uma atividade física de caminhada livre de acordo com a percepção do esforço à atividade física que eles adquiriram durante os seis meses.

Os dados foram apresentados com média \pm desvio padrão. A distribuição das variáveis foi avaliada pelo teste de normalidade Shapiro Wilk. Para a comparação entre os valores basais pré-treinamento e os valores pós-treinamento foi utilizado o teste t-student para medidas pareadas. $O$ valor de $P<0.05$ foi considerado estatisticamente significante.

\section{RESULTADOS}

Nove pacientes concluíram o PRC, apenas dois pacientes abandonaram o programa, um devido a motivos pessoais e o outro devido a internação hospitalar de longo período por infecção respiratória.

A média de idade dos pacientes foi de 66 anos (50 a 82 anos) e houve predominância do sexo masculino (55,6\%). Não houve alteração significativa no IMC após o treinamento $(27,1 \pm 2,8$ vs. $27,8 \pm 3,2 \mathrm{~kg} /$ $\left.\mathrm{m}^{2} ; \mathrm{P}=0,057\right)$. O tempo médio entre a cirurgia e o início do treinamento foi de $11 \pm 6$ meses. Não ocorreu nenhum evento adverso nos pacientes durante as sessões de treinamento.

Seis meses de PRC não modificou os níveis de $\mathrm{HDL}$, $L D L$, triglicerídeos, glicemia e insulina, porém houve um aumento do colesterol total e uma redução do nível de hemoglobina glicada (tabela 1).

A força muscular avaliada pelo teste de 1-RM apresentou um ganho significativo tanto para membros superiores $(9,77 \pm 4,14 \mathrm{~kg}$ vs. $12,55 \pm 4,90 \mathrm{~kg} ; \mathrm{P}=0,030$ ) (figura $1 \mathrm{~A}$ ) quanto para membros inferiores $(19,33 \pm 9,34 \mathrm{~kg}$ vs. $24,44 \pm 9,12 \mathrm{~kg} ; \mathrm{P}=0,038)$ (figura $1 \mathrm{~B})$. No TC6M, os pacientes alcançaram uma distância percorrida significativamente maior após o treinamento [(459 \pm 94 metros vs. $547 \pm 83$ metros $(P=0,020)$ ] (figura 1C).

Não houve variações significativas no diâmetro da artéria braquial e na vasodilatação mediada pela nitroglicerina entre os valores basais e após seis meses de PRC (tabela 1). Entretanto, houve uma melhora de 3,5\% no valor percentual da FMD após seis meses de PRC $(P=0,026)$ (figura 1D). O PRC executado durante seis meses reverteu a disfunção endotelial de dois pacientes, valores individuais do \%FMD apresentados na tabela 2 antes e depois do PRC.

Tabela 1. Parâmetros metabólicos e reatividade braquial antes e depois de seis meses do programa de reabilitação cardíaca.

\begin{tabular}{c|c|c|c}
\hline Parâmetros & Antes do PRC & Depois do PRC & $\mathbf{p}$ \\
\hline Colesterol total, mg/dL & $162 \pm 31$ & $195 \pm 39$ & 0,012 \\
\hline HDL, mg/dL & $39 \pm 8$ & $45 \pm 9$ & 0,056 \\
\hline LDL, mg/dL & $94 \pm 29$ & $109 \pm 36$ & 0,298 \\
\hline Triglicerídeos, mg/dL & $166 \pm 35$ & $150 \pm 26$ & 0,676 \\
\hline Glicemia, mg/dL & $110 \pm 48$ & $116 \pm 39$ & 0,283 \\
\hline Hemoglobina glicada, \% & $6,74 \pm 1,64$ & $6,26 \pm 1,62$ & 0,028 \\
\hline Insulina, $\mu \mathrm{U} / \mathrm{mL}$ & $8,78 \pm 4,60$ & $8,93 \pm 5,77$ & 0,903 \\
\hline Diâmetro, $\mathrm{mm}$ & $4,11 \pm 0,53$ & $4,23 \pm 0,58$ & 0,261 \\
\hline NMD, \% & $18,31 \pm 8,16$ & $20,31 \pm 7,72$ & 0,223 \\
\hline
\end{tabular}

Dados apresentados como media \pm desvio padrão. PRC = programa de reabilitação cardíaca; $\mathrm{NMD}$ = dilataçẫo mediada pela nitroglicerina (do ingl. nitroglycerin-mediated dilation). 
Tabela 2. Valores individuais do \%FMD antes e depois do programa de reabilitação cardíaca.

\begin{tabular}{c|c|c}
\hline Pacientes & Antes do PRC (\%) & Depois do PRC (\%) \\
\hline 1 & 5,05 & 6,04 \\
\hline 2 & 14,92 & 16,67 \\
\hline 3 & 3,06 & 7,27 \\
\hline 4 & 6,69 & 8,19 \\
\hline 5 & 5,90 & 14,51 \\
\hline 6 & 2,31 & 13,80 \\
\hline 7 & 4,42 & 6,70 \\
\hline 8 & 4,72 & 5,17 \\
\hline 9 & 10,09 & 10,81 \\
\hline Média & 6,35 & 9,91 \\
\hline Desvio padrão & 3,92 & 4,19 \\
\hline
\end{tabular}

Dados apresentados em valores percentuais. PRC = programa de reabilitação cardíaca; \%FMD = dilatação mediada pelo fluxo (do ingl. flow-mediated dilation).

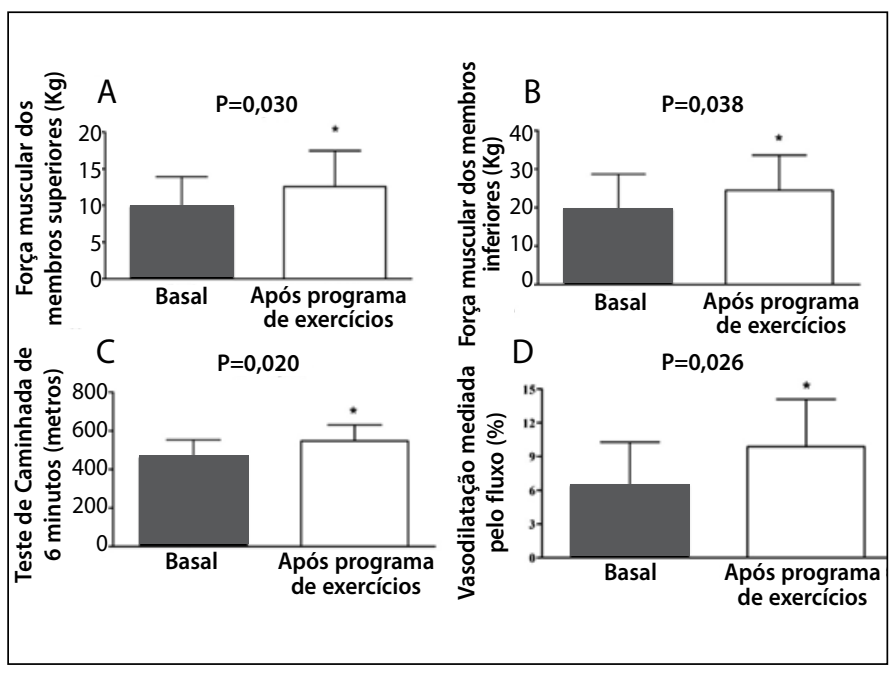

Figura 1. (A) Aumento da força muscular dos membros superiores após seis meses de PRC; (B) Aumento da força muscular dos membros inferiores após seis meses de PRC; (C) Distância percorrida antes e depois de seis meses de PRC; (D) Dilatação mediada pelo fluxo [Flow-mediated vasodilation (\%FMD)] na artéria braquial antes e depois de seis meses de PRC. * $P<0,05$.

\section{DISCUSSÃO}

No presente estudo, o treinamento físico de exercícios combinados realizados em seis meses consecutivos no programa de reabilitação cardíaca ajudou a restaurar a função endotelial como mostrado através da melhora da função vasomotora dependente do endotélio em pacientes sedentários submetidos à cirurgia de revascularização do miocárdio na fase tardia da reabilitação. Além disso, os pacientes apresentaram um ganho de força muscular em membros superiores e inferiores e uma melhora na capacidade funcional. Entretanto, o programa de reabilitação cardíaca por seis meses consecutivos não melhorou o perfil lipídico dos pacientes.

Recentemente, um estudo se propôs a avaliar os efeitos de drogas anti-agregantes plaquetárias sobre a função endotelial através da técnica FMD em pacientes com síndrome coronariana aguda submetidos à angioplastia com stent ${ }^{18}$. Entretanto, programas de reabilitação cardíaca são aceitos como uma intervenção terapêutica não farmacológica para o tratamento de pacientes após infarto agudo do miocárdio e/ou que foram submetidos à $\mathrm{CRM}^{19}$, além de melhorar a função endotelial na circulação coronária ${ }^{20}$ e periférica ${ }^{4,21,22}$. Em associação, ambos os tratamentos podem ajudar a diminuir as taxas de morbidade e mortalidade destes pacientes.
De acordo com nossos resultados, em pacientes com doença coronariana, não submetidos à intervenção cirúrgica, Gokce et al. ${ }^{21}$ observaram, após programa de exercícios, melhora na função vasomotora dependente do endotélio na artéria braquial, sem variações significantes na função vasomotora independente do endotélio.

$\mathrm{O}$ atual estudo aplicou um treinamento combinado de exercícios aeróbios e exercícios resistidos em seis meses de treinamento. Outros estudos já haviam comprovado uma melhora na função endotelial independente do tipo de treinamento (aeróbio, resistido ou combinado - aeróbio e resistido), do tempo empregado (um mês, três meses ou seis meses) e independente se a execução do treinamento foi realizada com instruções para a realização de exercícios em casa (home-based cardiac rehabilitation) ou se em um centro de reabilitação cardíaca convencional ${ }^{4,20}$. Vona et al. ${ }^{4}$ relatam que os benefícios na função endotelial vascular periférica adquiridos no treinamento podem ser perdidos em apenas um mês cessado o treinamento.

O TC6M tem sido utilizado para avaliar a capacidade funcional de pacientes em PRC 15,23. Estudos comprovaram a viabilidade e a segurança do teste num curto período após o paciente ter se submetido à CRM, mostrando que estes pacientes obtiveram uma melhora de 31\% na distância percorrida após a aderência a um PRC comparado ao valor basal antes da cirurgia ${ }^{15}$, sendo que esta melhora deve ser considerada um real benefício na capacidade funcional como uma consequência da intervenção terapêutica e não como uma variabilidade inter-teste ${ }^{24}$. No atual estudo, após longo período após a CRM (fase tardia da reabilitação - fase III), os pacientes apresentaram um aumento na distância percorrida no TC6M após o treinamento no PRC.

A melhora no desempenho da distância percorrida é um importante fator, pois caminhar em baixa velocidade é um componente de fragilidade e está associada com fraqueza muscular e redução de mobilidade ${ }^{16}$. Nosso estudo sugere que o ganho significativo de força muscular, especialmente em membros inferiores, adquirido com os exercícios resistidos pode ter contribuído para a melhora na distância percorrida no TC6M associados com o exercício aeróbio. Além disso, Lee et al. ${ }^{25}$ mostraram que a fragilidade é um significante preditor independente para a mortalidade e para internação prolongada após a cirurgia cardíaca. Portanto, exercícios resistidos estão sendo tidos como exercícios fundamentais em PRCs.

Não foi observado uma melhora no perfil lipídico dos pacientes nestes seis meses consecutivos de treinamento, porém o número de pacientes em nossa amostra foi pequeno para a análise. Kim et al. ${ }^{26} \mathrm{ob}-$ servaram que não houve diferença significativa no perfil lipídico quando comparado dois grupos: um com caminhada usual e o outro com caminhada com alta velocidade durante seis semanas em pacientes que sofreram intervenções percutâneas coronarianas. Vona et al.4 não observaram melhora significante no perfil lipídico em pacientes com infarto agudo do miocárdio que realizaram treinamento com exercícios aeróbios, resistidos ou com treinamento combinado após um mês. Em pacientes coronários submetidos a seis meses de PRC, Pavy et al. ${ }^{27}$ observaram um aumento do HDL em 202 pacientes divididos em três diferentes grupos de acordo com a faixa etária. Lee et al. ${ }^{19}$ observaram redução do colesterol total e aumento no HDL em pacientes após infarto agudo do miocárdio ou submetidos à CRM que participaram de um PRC em ambiente hospitalar. Entretanto, tal melhora não foi observada no outro grupo deste estudo que realizaram a reabilitação cardíaca em casa, ambos em três meses. Desta forma, observa-se que o tempo de PRC empregado e a forma de aplicação (convencional ou domiciliar) parecem poder interferir na melhora do perfil lipídico.

Como limitação do estudo verificamos que a amostra do estudo foi composta por poucos pacientes, pois a procura pelo serviço de 
reabilitação cardíaca é baixa, a adesão ao serviço é baixa e a manutenção destes pacientes por longo período em treinamento é uma tarefa difícil. Em nosso estudo de onze participantes, ocorreram duas perdas devido à ocupação de trabalho e devido a uma internação hospitalar por infecção respiratória.

\section{CONCLUSÃO}

Concluiu-se com este estudo que o treinamento físico proporcionado no PRC num período de seis meses consecutivos ajudou a melhorar a função endotelial vascular e a capacidade funcional de pacientes sedentários que foram submetidos à cirurgia de revascularização do miocárdio isoladamente na fase tardia da reabilitação cardíaca, sem que houvesse modificações no perfil lipídico dos mesmos.

\section{AGRADECIMENTOS}

Os autores agradecem a Associação de Caridade Santa Casa do Rio Grande, RS, pelo suporte financeiro dado a esta pesquisa.

Todos os autores declararam não haver qualquer potencial conflito de interesses referente a este artigo.

\section{REFERÊNCIAS}

1. Leon AS, Franklin BA, Costa F, Balady GJ, Berra KA, Stewart KJ, et al. Cardiac rehabilitation and secondary prevention of coronary heart disease. An American Heart Association Scientific Statement From the Council on Clinical Cardiology (Subcommittee on Exercise, Cardiac Rehabilitation, and Prevention) and the Council on Nutrition, Physical Activity, and Metabolism (Subcommittee on Physical Activity), in Collaboration With the American Association of Cardiovascular and Pulmonary Rehabilitation. Circulation. 2005;111:369-376

2. Taylor RS, Brown A, Ebrahim S, Jolliffe J, Noorani H, Rees K, et al. Exercise-based rehabilitation for patients with coronary heart disease: systematic review and meta-analysis of randomized trials. Am J Med. 2004;116(10):682-97.

3. Vuckovic KM, Piano MR, Phillips SA. Effects of exercise interventions on peripheral vascular endothelial vasoreactivity in patients with heart failure with reduced ejection fraction. Heart Lung Circ. 2013;22(5):328-40.

4. Vona M, Codeluppi GM, lannino T, Ferrari E, Bogousslavsky J, Von Segesser LK. Effects of different types of exercise training followed by detraining on endothelium-dependent dilation in patients with recent myocardial infarction. Circulation. 2009;119(12):1601-8.

5. Luk TH, Dai YL, Siu CW, Yiu KH, Chan HT, Lee SW, et al. Effect of exercise training on vascular endothelial function in patients with stable coronary artery disease: a randomized controlled trial. Eur J Prev Cardiol. 2012:19(4):830-9.

6. Morikawa Y, Mizuno Y, Harada E, Katoh D, Kashiwagi Y, Morita S, et al. Aerobic interval exercise training in the afternoon reduces attacks of coronary spastic angina in conjunction with improvement in endothelial function, oxidative stress, and inflammation. Coron Artery Dis. 2013;24(3):177-82.

7. Anagnostakou V, Chatzimichail K, Dimopoulos S, Karatzanos E, Papazachou O, Tasoulis A, et al. Effects of interval cycle training with or without strength training on vascular reactivity in heart failure patients. J Card Fail. 2011;17(7):585-91.

8. Credeur DP, Mariappan N, Francis J, Thomas D, Moraes D, Welsch MA. Vasoreactivity before and after handgrip training in chronic heart failure patients. Atherosclerosis. 2012;225(1):154-9.

9. Shechter M, Shechter A, Koren-Morag N, Feinberg MS, Hiersch L. Usefulness of brachial artery flow-mediated dilation to predict long-term cardiovascular events in subjects without heart disease. Am J Cardiol. 2014;113(1):162-7.

10. Lerman A, Zeiher AM. Endothelial function: cardiac events. Circulation. 2005;111(3):363-8.

11. Leung FP, Yung LM, Laher I, Yao X, Chen ZY, Huang Y. Exercise, vascular wall and cardiovascular diseases: an uptade (Part 1). Sports Med. 2008;38 (12):1009-24

12. Marsh SA, Coombes JS. Exercise and the endothelial cell. Int J Cardiol. 2005;99 (2):165-9.

13. Oldridge NB, Guyatt G, Fischer M, Rimm AA. Cardiac rehabilitation after myocardial infarction: combined experience of randomized clinical trials. JAMA. 1988;260 (7):945-50.
14. O'Connor G, Burng Y, Yusuf S, Goldhaber SZ, Olmstead EM, Paffenberger RS Jr, et al. An overview of randomized trials of rehabilitation with exercise after myocardial infarction. Circulation. 1989;80(2):234-44.

15. Fiorina C, Vizzardi E, Lorusso R, Maggio M, De Cicco G, Nodari S, et al. The 6-min walking test early after cardiac surgery. Reference values and the effects of rehabilitation programme. Eur J Cardiothorac Surg. 2007;32(5):724-9.

16. Zielińska D, Bellwon J, Rynkiewicz A, Elkady MA. Prognostic value of the six-minute walk test in heart failure patients undergoing cardiac surgery: a literature review. Rehabil Res Pract. 2013;2013:965494.

17. Correti MC, Anderson TJ, Benjamin EJ, Celermajer D, Charbonneau F, Creager MA, et al. Guidelines for the ultrasound assessment of endothelial-dependent flow-mediated vasodilation of the brachial artery. J Am Coll Cardiol. 2002;39(2):257-65.

18. Schnorbus B, Daiber A, Jurk K, Warnke S, König J, Krahn U, et al. Effects of clopidogrel, prasugrel and ticagrelor on endothelial function, inflammatory and oxidative stress parameters and platelet function in patients undergoing coronary artery stenting for an acute coronary syndrome. A randomised, prospective, controlled study. BMJ Open. 2014;4:e005268

19. Lee KW, Blann AD, Jolly K, Lip GYH. Plasma haemostatic markers, endothelial function and ambulatory blood pressure changes with home versus hospital cardiac rehabilitation: the Birmingham Rehabilitation Uptake Maximisation Study. Heart. 2006;92(12):1732-8.

20. Hambrecht R, Wolf A, Gielen S, Linke A, Hofer J, Schuler G. Effect of exercise on coronary endothelial function in patients with coronary artery disease. N Engl J Med. 2000;342(7):454-60.

21. Gokce N, Vita JA, Bader DS, Sherman DL, Hunter LM, Holbrook M, et al. Effect of exercise on upper and lower extremity endothelial function in patients with coronary artery disease. Am J Cardiol. 2002;90(2):124-7.

22. Edwards DG, Schofield RS, Lennon SL, Pierce GL, Nichols WW, Braith RW. Effect of exercise training on endothelial function in men with coronary artery disease. Am J Cardiol. 2004;93(3):617-20.

23. Opasich C, De Feo S, Pinna GD, Furgi G, Pedretti R, Scrutinio D, et al. Distance walked in the 6-minute test soon after cardiac surgery. Chest. 2004;126(6):1796-801.

24. Opasich C, Pinna GD, Mazza A, Febo O, Riccardi PG, Capomolla S, et al. Reproducibility of the six-minute walking test in patients with chronic congestive heart failure: practical implications. Am J Cardiol. 1998;81(12):1497-500

25. Lee DH, Buth KJ, Martin B, Yip AM, Hirsch GM. Frail patients are at increased risk for mortality and prolonged institutional care after cardiac surgery. Circulation. 2010;121(8):973-8.

26. Kim C, Kim BO, Lim KB, Kim YJ, Park YB. The Effect of Power-walking in Phase 2 Cardiac Rehabilitation Program. Ann Rehabil Med. 2012; 36(1):133-40.

27. Pavy B, Caillon M. Effects of a cardiac rehabilitation programme in coronary patient according to age Ann Cardiol Angeiol (Paris). 2012;61(5):338-44. 